\title{
DESIGN AND CONSTRUCTION OF MASSIVE DIGITAL ORTHOPHOTO MAP DATABASE IN CHINA
}

\author{
YANG Mei, LIU Jianjun, ZHANG Yuanjie, LI Xuemei
}

National Geomatics Centre of China, 100830 Beijing, China (yangmei, liujianjun, zhangyj, lixm)@nsdi.gov.cn

\author{
Commission IV, WG IV/2
}

KEY WORDS: Digital Orthophoto Map, DOM, Image, Massive data, Database, Design, Construction

\begin{abstract}
:
Digital orthophoto maps have great advantages of high precision, plentiful information, fine intuition and visualization and convenient acquisition, as one of the most important part of national spatial data infrastructure (NSDI), and a type of required data resources in geo-spatial industry. Within last few years, large volume of high-resolution remotely sensed images have become available, obtained by various remotely sensed platforms. The acquired remotely sensed images continuously increasing by exponential order have brought a series of problems about storage, management, distribution and applications of massive image data. The objectives of the following research is to investigate how to design and construct national digital orthophoto map database which is mainly constituted by domestic remotely sensed images. In this paper, according to technical characteristics of national fundamental geo-information image database, we analyzed and then put forward the demands and functions of multi-source and massive database of digital orthophoto maps, and constructed an example of DOM database primarily on the basis of domestic remoted images such as ZY-3 and TH-1.
\end{abstract}

\section{INTRODUCTION}

Digital orthophoto maps (DOM) have great advantages of high precision, plentiful information, fine intuition and visualization, and convenient acquisition, as one of the most important part of national spatial data infrastructure (NSDI)(Tian, 2006), and a type of required data resources in geo-spatial industry. In the continuous maintenance of fundamental geo-information DOM is not only one key part of national fundamental geo-spatial database, and also significant information resource of other geospatial data such as digital line graphic, digital elevation model and digital cartographic data (Liao, 2006). During the process of national economy construction and social development service, DOM could provide a lot of straightforward and convenient image applications for land cover classification, territory monitoring, resources and environment, city investigation, urban planning, forest management, agriculture assessment, disaster prevention and relief, public mapping service, and so on (Li, 2003; Wang, 2003).

In the past several years with rapid development of computer technology and spatial science, the sources of remotely-sensed images have been getting richer and richer, and the image quality has also been improved obviously. High-resolution remotely-sensed images from different types of platforms are transferred to earth like rain drops (Yang, 2014; Dong, 2012). Remotely-sensed data size is growing by exponential order these years, which stressed a series of problems about storage, management, distribution, service and application of massive image data. On the other hand, dynamic updating mode of national geo-information database has been adopted to adapt to the freshness demands from different fields, with rapid development of the whole society. Nowadays, either DLG, or DEM, or DOM, or cartographic data, are being updated in the speed of several ten times higher than before. According to the characteristics of high resolution and updating speed, large data size and wide-spread applications, we need to establish an integrated image database, to satisfy urgent requirement about the storage, management, distribution and application of multiresolution, multi-sense and multi-source digital orthophoto images, to supply a steady and reliable platform of image data for large surveying and mapping projects, public service and emergency support.

\section{DEMANDS OF DOM DATABASE}

\subsection{Objects of database construction}

The main object of constructing national DOM database is to organize and to manage multi-source, multi-resolution and multi-tense centralized or distributed digital orthophoto maps on the basis of standard image data with identical code and unified format, to implement the scan, browse and application quickly and seamlessly, from whole to partial, from overall to detailed and from low-resolution to high-resolution. The database should consists of several functions such as spatial query, roving and distribution, and could also be integrated with other database systems to supply a unified and efficient platform.

\subsection{Demands of construction}

As a large national spatial database with the key contents of digital orthophoto maps, the main demands of construction are as follows.

\subsubsection{Data management}

It is quite required to manage multi-source, multi-resolution and multi-tense centralized or distributed digital orthophoto maps and corresponding metadata. Horizontally, images of the whole 
country should be organized as a logically seamless mosaic. Vertically, multi-tense image data could be overlaid and combined according to identical and consistent spatial coordinates.

\subsubsection{Data application}

We need to implement efficient retrieval of massive image data, including spatial retrieval and attribute retrieval, to implement the scan, browse and application quickly and seamlessly, from whole to partial, from overall to detailed and from lowresolution to high-resolution, and to implement complicated application and service integrated with other systems.

\subsubsection{Data distribution}

It is important for remote users to implement a series of functions query, inquiry, ordering and downloading through local area network or wide area network.

\section{FUNCTION DESIGN}

The basic requirement of national DOM database is "being placed safely, being seen clearly, being inquired quickly and being taken away comfortably". Combined with the future practical applications, nine aspects has been considered and proposed while designing the main database functions, which is shown in Fig. 1.

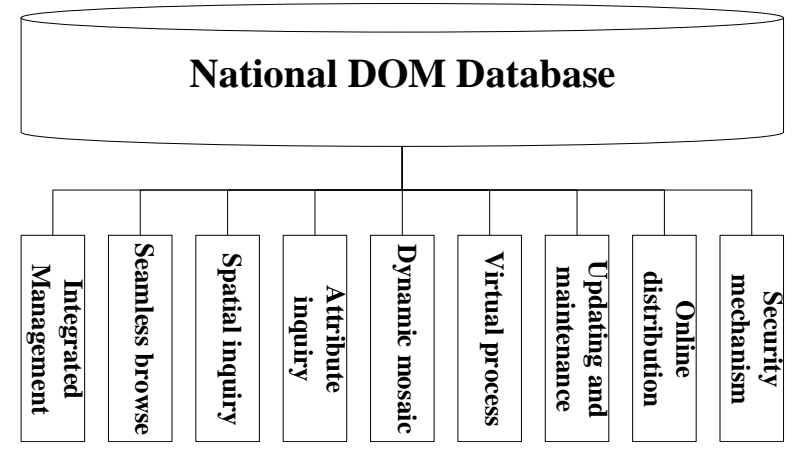

Figure 1. Function structure of national DOM database

\subsection{Integrated management}

One of the most important objects of national DOM database is integrated management, which means to organize and to manage multi-source, multi-resolution and multi-tense centralized or distributed digital orthophoto maps. Images are stored as records in the image database, each record corresponds to an image. The recording table is composed of several attributes about spatial index data, coordinates of central point, displaying resolution's control extent, pixel size extent, image type tag, control factor of displaying order, grouping label, etc. Using spatial index data of the recording table, we can directly access each image's specific information, such as thumbnail, data source, image attributes, geographic extent, spatial reference, pixel value's statistics, situations of pyramids and colour tables, real-time processing function chain, etc.

\subsection{Seamless browse}

Seamless browse means an overall integrated and visual display of images all around the country, and an implementation of scan and browse quickly and seamlessly, including manual roaming, roaming in a designed path, zooming in, zooming out, crosslayer browse, full-extent display, 1:1 display, etc. Due to different sources of national DOM images, the spatial references adopted may be not concordant, for example, some use geographic coordinate system, and others use Gauss Kruger zoning projection coordinate system, though. Consequently, a database interface oriented to different spatial references should be designed.

\subsection{Spatial inquiry}

Through spatial position inquiry in the national DOM database, we can get access to specific images related to a particular point, line or polygon, achieve the inquired results quickly and show their thumbnails and attributes.

\subsection{Attribute inquiry}

According to attribute information such as data source, updating time, spatial resolution and spatial position, we can inquire image records in accordance with the specified demands using structured query language, achieve the inquired results quickly and show their thumbnails and attributes.

\subsection{Dynamic mosaic}

The dynamic mosaic method has been adopted to display multisource, multi-resolution and multi-tense digital orthophoto images synthetically, which could implement a desired mosaic effect and also could save a lot of time and disk space for massive image mosaic process.

\subsection{Virtual process}

The original pixel values should not be processed and changed to ensure the consistence and certainty of original digital orthophoto maps. Therefore, various types of virtual image products could be derived from the basic images, without intermediate results.

\subsection{Updating and maintenance}

It is rather necessary to maintain the currency of image data (Xie, 2011). Adding new digital orthophoto images to national DOM database in the first time is one of the most important step for later supply and application. The main functions are constituted with import, export, deletion and update. Import operations include several ways such as single sheet, batch sheets and following addition. Export operations include several ways such as database, layer, sheet and spatial extent (coordinates). Database maintenance includes deletion and updating, in which updating function should support and satisfy image updating in any local area (Huang, 2011).

\subsection{Online distribution}

The contents of online distribution service of digital orthophoto maps mainly contain accepting a data inquiry request by $\mathrm{B} / \mathrm{S}$ structure, calling out inquired results and transferring the results to users through internet. 


\subsection{Security mechanism}

In consideration of security of the whole database, we adopt a mainstream database management system software: Oracle, which could authorize visiting and operating for users of different grades and groups, and could validate users' authority.

\section{DATABASE DESIGN}

\subsection{Spatial reference}

In order to implement integrated management and seamless browse of the DOM database covering the whole country, the database adopts China Geodetic Coordinate System 2000 (CSCS2000) and National Vertical Datum 1985. Images are dynamically projected to CSCS2000 coordinates.

\subsection{Data organization}

The DOM database is consisted of different types of files, such as digital orthophoto maps, image pyramids, image processing recording aux files, image datasets files, and dynamic mosaic overview files.

Entity files such as digital orthophoto maps, image pyramids, image processing recording aux files, etc. are stored in a changeless physical position and organized by an order of "source-year-month", in a basic unit of months. These files could be placed in concentration or distribution pattern.

Mosaic dataset and its overview files are stored in SDE database. Whether the entity files, mosaic dataset or overview files, their physical position could be adjusted for possible upgrade and expansion of the database in the future.

\subsection{Design of database structure}

\subsubsection{Logic design}

The logic structure of DOM database system includes four levels: data layer, management layer, service layer and user layer, which is shown in Figure 2.

1) Data layer is consisted of a series of files, including digital orthophoto maps, overview data, metadata and relational tables. 2) Management layer contains a set of managing functions, including import, export, addition, deletion, modification, inquiry and update of images.

3 ) Service layer contains a series of service functions such as seamless browse, inquiry and distribution.

4) User layer is constituted by a large number of users connected to images and corresponding services through local area network or wide area network.

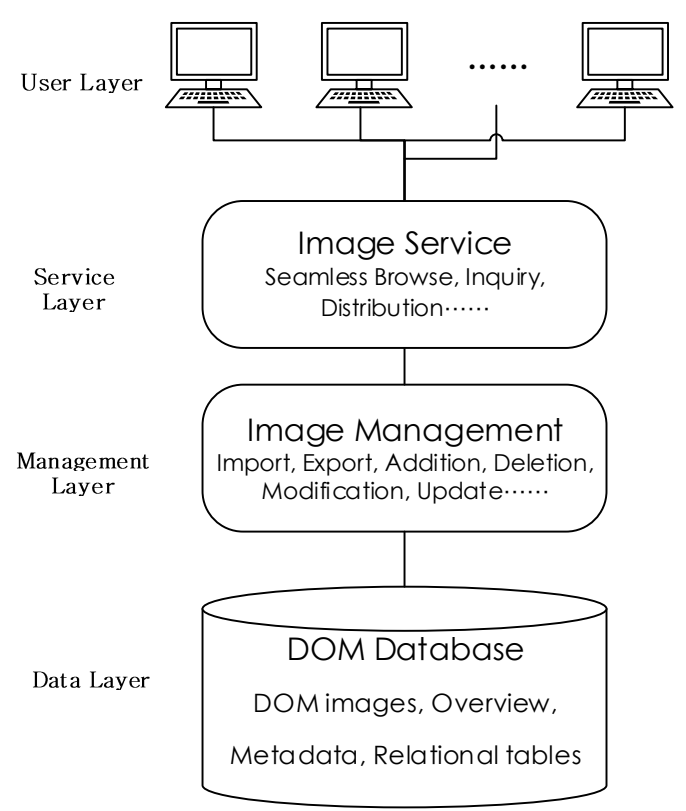

Figure 2 The logic structure chart of DOM database system

\subsubsection{Table design}

In the DOM database, four relational tables are mainly designed to manage static and dynamic information of DOM images, which are the total table, image type table, size classifying table and $\log$ table. The table structures and their relationships between each other are as seen in Figure 3. Additionally, all the tables could be extended for more attributes according to further and deeper demands of management and application.

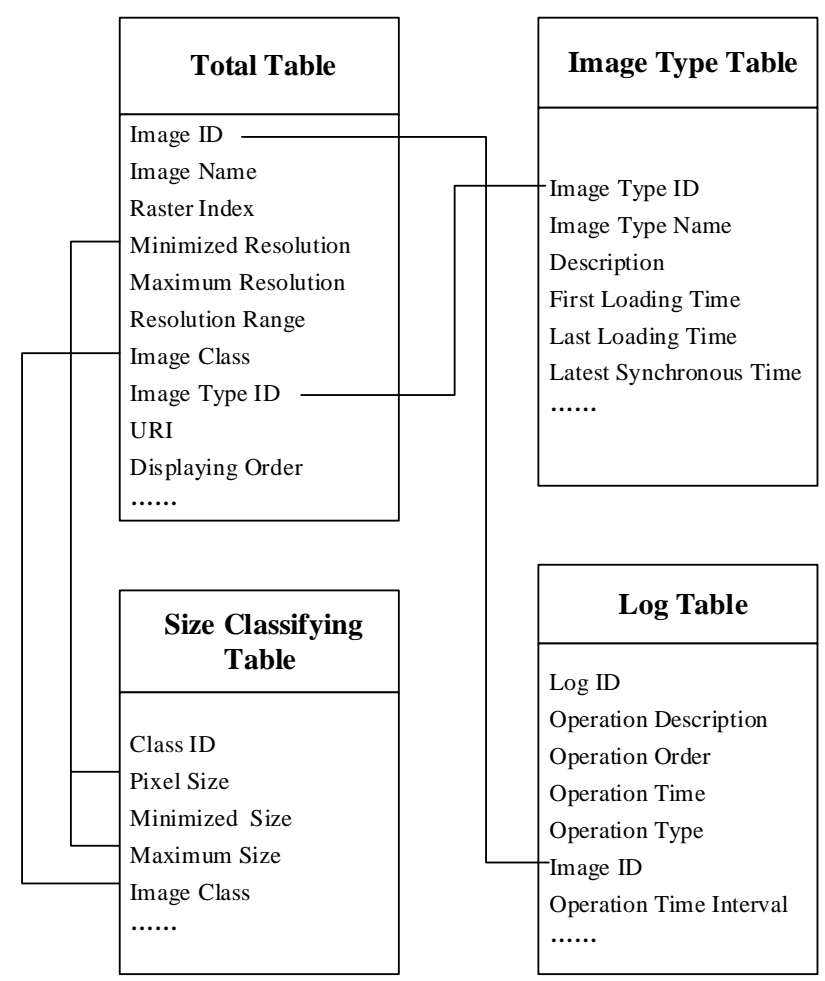

Figure 3 The chart of DOM database tables 


\section{EXPERIMENT}

The national DOM database of China was established using domestic satellite images such as ZiYuan 3 and TianHui 1 from 2011 to 2014, which consists of 33531 images and covers $96.21 \%$ of the whole mainland. The total data size is about twenty TB.

Firstly, the images are organized, normalized, inspected by automatic algorithm combined with human interaction, then the pyramids build, image statistics and projection unity are carried out to keep in accordance with specified standards, in order to make sure the data being imported to DOM database.

Multithread technology is employed while images are imported, the bandwidth of network interface is up to $1000 \mathrm{MB}$ per second. Through document storage combined with database index, images could be imported into the national database in several days.

The image database could be browsed based on a set of layers of pyramids and overviews. Pyramid building is a sort of image storing technology which reduces the single image raster's resolution by $2: 1$ ratio, while overview building is directed to the whole dynamic mosaic image ,and its resolution is reduces by $n: 1 \quad(n>=2)$ ratio. According to current operations (zoom in, zoom out, roam, etc.), necessary view extent and displaying resolution are calculated and matched, the final results are displayed on the screen. By this way, seamless display effects could be implemented around the whole country, as well as continuous roaming, leaping roaming, fast zooming, 1:1 zooming et al. additionally, attribute information could be inquired by metadata management system and geographic information could be inquired by spatial analysis module, the inquired results could be used for subset extraction and distribution service in the next step. When basic images change, including adding, deleting and replacing, the database should be updated immediately on the basis of the old foundation.

\section{CONCLUSION}

Focusing on the problems of storage, management, browse and updating of digital orthophoto maps and using new raster data management model(ESRI, 2013) and spatial database technology(Guo, 2007), we implemented distributed storage, centralized management, seamless browse and fast updating of national massive multi-source database of digital orthophoto maps, which would help efficient application and information excavation of digital orthophoto maps, and avoid repeated construction in the field of geography and spatial information. In the next step, we will deeply develop optimized functions corresponding to image database management and service, and furtherly study the technologies about seamless browse, inquiry and query, distribution service, updating and maintenance, in order to increase the efficiency and technical level of massive and multi-source image management.

\section{ACKNOWLEDGEMENTS}

We would like to give our acknowledgements to National Fundamental Geo-information Database Dynamic Updating Project for the strong support in this study.

\section{REFERENCES}

Dong J, Huang Y, Dong P. 2012. Research on Management of Large Quantities of Image Data Based on Mosaic Dataset. Infrared, 33(10), pp.30-32.

ESRI. Raster and Images [EB/OL]. [2013-08-

25].http://resources.arcgis.com/zh-cn/help/main/10.1/index.html.

Guo C H, Qi Q W, Zou X P, et al. 2007. A Study of the

Construction of ArcSDE-based Ecological and Environmental

Database of Yunnan Boundary Region. Bulletin of Sureveying and Mapping , (3), pp. 53-56.

Huang F P. 2011.Design and Implement of Massive Remote Sensing Image Management System. Shanghai. East China Normal University, pp.14-19.

Li J W, Liao A P, Zhu W. 2003. Ideas of National Basic Imagery Database Construction and Its Progress. Geomatics World, 1(2), pp. 30-34.

Liao A P, Yan R H, Tang H. 2006. Design \& Establishment of National Fundamental Multisource DOM Database. Geomatics World, (4), pp. 10-14.

Tian M Y, Lu X S, Wang D, et al, 2006. Study of DOM Image Database Establishment Based on Oracle. Bulletin of Surveying and Mapping, (6), pp. 13-16.

Wang M, Gong J Y, Li D R. 2003. Design and Implementation of Large-Scale Image Database Management System. Geomatics and Information Science of Wuhan University, (3), pp. 294-299.

Xie Y. 2011. Research on the Storing and Organizing Structure of Massive Remote Sensing Image Data. Kaifeng. The Graduate School of Henan University, pp.20-24.

Yang M, Liu J J, Li X M, et al. 2014. A Preliminary Study on Constructing DOM Database Using ArcGIS Mosaic Dataset. Geomatics World, 21(1), pp. 33-36. 